\title{
SUSY SEARCHES WITH ATLAS
}

\author{
Matthias Hamer, ${ }^{a}$ \\ On behalf of the ATLAS Collaboration \\ II. Physikalisches Institut, Georg-August-University Göttingen, Germany \\ Abstract. These proceedings summarize seven searches for supersymmetry with \\ the ATLAS detector at the LHC. In each analysis, the full $\sqrt{s}=8 \mathrm{TeV}$ data \\ set, corresponding to an integrated luminosity of approximately $20 \mathrm{fb}^{-1}$, has been \\ used. No significant excess above the Standard Model expectation is observed.
}

\section{Introduction}

The concept of supersymmetry (SUSY) [1] is one of the most promising and therefore most prominent extensions to the Standard Model of Elementary Particle Physics (SM). Despite already known to be broken, by introducing a symmetry between fermionic and bosonic degrees of freedom, SUSY can provide a natural solution to the SM hierarchy problem, a unification of the SM gauge couplings at a high energy scale and an excellent candidate for dark matter [1].

At the LHC [2] it is possible to test SUSY at the TeV scale. With more than 100 soft SUSY breaking parameters a huge number of experimental signatures is possible. In these proceedings, seven searches for supersymmetry with the ATLAS detector [3] are presented.

\section{Inclusive Searches for Squarks and Gluinos}

If the squarks and the gluino are not too heavy, the direct production of these dominates the total SUSY cross section at the LHC. Assuming R-parity conservation, they are produced in pairs and final states with jets and missing transverse energy $\left(\mathrm{E}_{T}^{\mathrm{miss}}\right)$ provide high sensitivity. In two recent searches $[4,5]$, the selection requires the existence of 2-10 jets, no leptons, and a significant amount of $\mathrm{E}_{T}^{\mathrm{miss}}$ in the event. Signal regions are defined by the jet multiplicity, the jet $p_{T}$, the effective mass and the amount of $\mathrm{E}_{T}^{\mathrm{miss}}$. All signal regions exhibit good agreement between data and the SM expectation. Figure 1 shows the derived limits in the CMSSM [1] and two simplified models for direct gluino production, where the gluino decays via an off-shell stop quark or gauginos.

\section{Searches for $3^{r d}$ Generation Squarks}

The "natural" stabilization of the Higgs boson mass is one of the motivations for scenarios in which the stop and sbottom masses are close to the electroweak scale. In a challenging scenario [6] the dominant decay of the stop is $\tilde{t} \rightarrow \tilde{\chi}_{1}^{0} c$. Assuming R-parity conservation, a search in two signal regions requires a high

\footnotetext{
${ }^{a}$ E-mail: matthias.hamer@cern.ch
} 

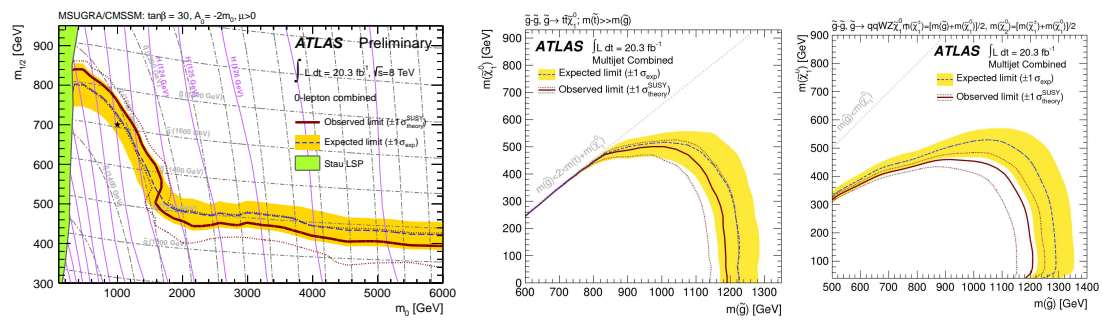

Figure 1: 95\% CL observed and expected exclusion simits in the CMSSM [4] and two simplified models for direct gluino production [5].

$p_{T}$ initial state radiation (ISR) jet, at least $220 \mathrm{GeV}$ of $E_{T}^{\text {miss }}$, the absence of isolated leptons and a minimum angle between $\mathrm{E}_{T}^{\text {miss }}$ and the ISR jet [6]. The two signal regions are then defined by requirements on the maximum or minimum number of additional jets, where in one signal region a low $p_{T}$ jet has to be identified as a c-jet. In both signal regions the data is compatible with the SM hypothesis, and a limit has been set in the $m_{\tilde{t}^{-}} m_{\tilde{\chi}_{1}^{0}}$ plane. Stops with a mass of up to $250 \mathrm{GeV}$, roughly, are excluded, as shown in Figure 2.

\section{Searches for Electroweak SUSY Production}

Scenarios with light electroweak gauginos and sleptons can improve the agreement between prediction and measurement of $(g-2)_{\mu}[8]$. In such scenarios, if the squarks and gluinos are too heavy to be produced at the LHC, the direct production of the electroweak gauginos and sleptons dominates the total SUSY cross section and final states with two or more leptons provide the best sensitivity. Searches for the direct production of two sleptons [7] and the direct production of a neutralino in association with a chargino [9] have been performed in final states with two or three light leptons. The target decay chains
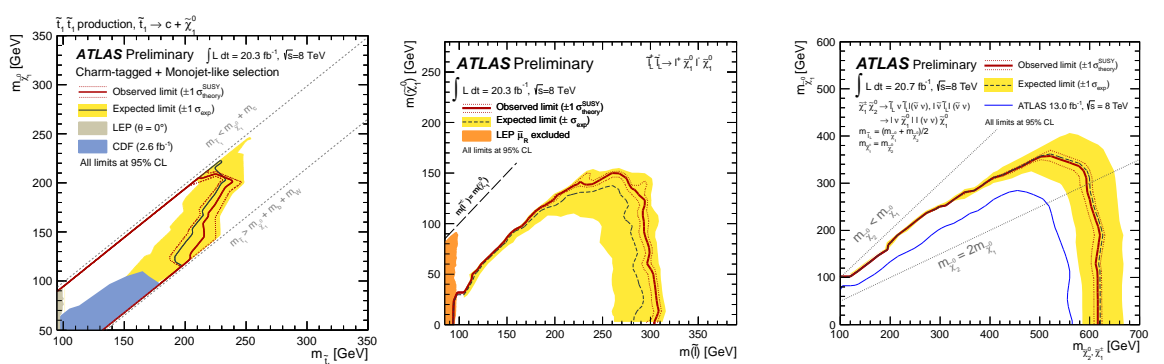

Figure 2: 95\% CL observed and expected exclusion limits in the direct searches for stops [6], sleptons [7] and electroweak gauginos [9]. 
are $\tilde{l} l \rightarrow \tilde{\chi}_{1}^{0} l \tilde{\chi}_{1}^{0} l$ and $\tilde{\chi}_{2}^{0} \tilde{\chi}_{1}^{ \pm} \rightarrow \tilde{\chi}_{1}^{0} l l \tilde{\chi}_{1}^{0} l \nu$, where the neutralino and chargino decays via sleptons. In addition to the existence of two or three light leptons in the final state, a high amount of $\mathrm{E}_{T}^{\text {miss }}$ and the absence of a $Z \rightarrow l l$ candidate is required. No evidence for physics beyond the SM is found, and limits have been set in simplified models as shown in Figure 2 .

\section{Searches for RPV and long-lived SUSY}

If SUSY is not considered as a candidate for dark matter, the nature of the lightest superpartner (LSP) is arbitrary and the LSP may be unstable, as is the second lightest superpartner (NLSP). If the LSP is still a neutralino, depending on the RPV couplings, final states with four or more leptons provide good sensitivity to such models. A search targeting a scenario with a gluino NLSP as well as a scenario with a wino-like NLSP has been performed, and no significant discrepancy between the observation and the SM expectation was found [10]. A neutralino LSP and a gluino NLSP with a mass of up to $1.5 \mathrm{TeV}$ as well as a wino-like NLSP with a mass of up to $750 \mathrm{GeV}$ could be excluded.

In AMSB-like models [1], where the LSP is stable, the NLSP may be semistable and charged. In such a scenario, a promising signature are disappearing tracks in the ATLAS Inner Detector. A search for such a model has been performed using a high $p_{T}$ ISR jet to tag events and using the number of hits in the ATLAS TRT to identify disappearing tracks [11]. No SUSY-like excess is found in this analysis, such that in an AMSB model a chargino NLSP with a mass of up to $530 \mathrm{GeV}$ and an half-life of up to $10 \mathrm{~ns}$ has been excluded.

\section{Summary}

Although no evidence for SUSY has been found yet in the searches performed by the ATLAS Collaboration, it remains a promising extensions to the SM. A large fraction of SUSY parameter space will be explored in the Run 2 of the LHC with an increased centre-of-mass energy and even higher luminosities.

[1] Kane, G.L. (ed.), Perspective on supersymmetry II

[2] L. Evans and P. Bryant (editors) 2008 JINST 3 S08001

[3] ATLAS Collaboration, 2008 JINST 3 S08003

[4] ATLAS Collaboration, ATLAS-CONF-2013-047

[5] ATLAS Collaboration, JHEP10(2013)130

[6] ATLAS Collaboration, ATLAS-CONF-2013-068

[7] ATLAS Collaboration, ATLAS-CONF-2013-049

[8] P. Bechtle et al., JHEP 1206 (2012) 098

[9] ATLAS Collaboration, ATLAS-CONF-2013-035

[10] ATLAS Collaboration, ATLAS-CONF-2013-036

[11] ATLAS Collaboration, arXiv:1310.3675, submitted to PRD 